\title{
ChemComm
}

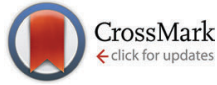

Cite this: Chem. Commun., 2015, 51,330

Received 13th October 2014, Accepted 5th November 2014

DOI: $10.1039 / c 4 c c 08072 f$

www.rsc.org/chemcomm

\section{Tuning intramolecular electron and energy transfer processes in novel conjugates of $\mathrm{La}_{2} \mathrm{CC}_{80}$ and electron accepting subphthalocyanines $\dagger$}

\author{
Lai Feng, ${ }^{\mathrm{ab}}$ Marc Rudolf, ${ }^{\mathrm{c}}$ Olga Trukhina, ${ }^{\text {de }}$ Zdenek Slanina, ${ }^{f}$ Filip Uhlik, ${ }^{g}$ Xing Lu, ${ }^{\text {h }}$ \\ Tomas Torres, ${ }^{* d e}$ Dirk M. Guldi*c and Takeshi Akasaka*bhij
}

\begin{abstract}
A series of two conjugates with $\mathrm{La}_{2} \mathrm{QC}_{80}$ and subphthalocyanine (SubPc) have been prepared and characterized by means of cyclic voltammetry, absorption, fluorescence, and femtosecond resolved transient absorption spectroscopy. The strong electron-donating character of $\mathrm{La}_{2} \mathrm{CC}_{80}$ is essential to power an intramolecular electrontransfer in the $\mathrm{La}_{2} \mathrm{CC}_{80}-\mathrm{SubPc}$ conjugates upon photoexcitation.
\end{abstract}

Mimicking photosynthesis has been of a great interest owing to the increasing need for an efficient and sustainable conversion of solar energy. ${ }^{1,2}$ One of the key factors in the reproduction of natural photosynthesis is to understand electron-transfer events between different electron donors and acceptors of the photosynthetic apparatus. Importantly, the nature of electron donors and acceptors determines the magnitude of electron transfer and, in turn, affects the overall yield of photosynthesis. Extensive studies have been carried out to design, to synthesize, and to probe electron donor and electron acceptor materials with improved performances in solar energy conversion. ${ }^{3,4}$ For photovoltaic

${ }^{a}$ College of Physics, Optoelectronics and Energy \& Collaborative Innovation Center of Suzhou Nano Science and Technology, Soochow University, 215006 Suzhou, China.E-mail:fenglai@suda.edu.cn

${ }^{b}$ Life Science Center of Tsukuba Advanced Research Alliance, University of Tsukuba, 305-8577 Tsukuba, Japan. E-mail: akasaka@tara.tsukuba.ac.jp

${ }^{c}$ Department of Chemistry and Pharmacy \& Interdisciplinary Center for Molecular Materials, Friedrich-Alexander-Universität Erlangen-Nürnberg, 91058 Erlangen, Germany.E-mail: dirk.guldi@fau.de

${ }^{d}$ Department of Organic Chemistry, Autonoma University of Madrid, 28049 Madrid, Spain.E-mail: tomas.torres@uam.es

${ }^{e}$ IMDEA Nanoscience, 9 Faraday, 28049 Madrid, Spain

${ }^{f}$ Department of Chemistry and Biochemistry, National Chung-Cheng University, 62117 Chia-Yi, Taiwan, Republic of China

${ }^{g}$ Department of Physical and Macromolecular Chemistry, Charles University in Prague, 6 Albertov, 12843 Praha 2, Czech Republic

${ }^{h}$ College of Materials Science and Engineering, Huazhong University of Science and Technology, 430074 Wuhan, China

${ }^{i}$ Foundation for Advancement of International Science, 305-0821 Tsukuba, Japan

${ }^{j}$ Department of Chemistry, Tokyo Gakugei University, 184-8501 Koganei, Japan

$\dagger$ Electronic supplementary information (ESI) available: Experimental details, complete characterizations of $\mathbf{1 a}, \mathbf{1} \mathbf{b}$, and selected transient absorption spectra. See DOI: $10.1039 / \mathrm{c} 4 \mathrm{cc} 08072 \mathrm{f}$ applications, fullerenes stand out among the myriad of electron accepting materials. ${ }^{5}$ Owing to their unique structural and redox features, fullerenes have been widely integrated into a wide facet of electron donor-acceptor systems. ${ }^{6}$ It is also well known that the use of empty fullerenes $\mathrm{C}_{60}$ and $\mathrm{C}_{70}$ as electron donors in molecular photovoltaics is limited by their poor electron-donating ability. The generation of radical cations of, for example, $\mathrm{C}_{60}$ or $\mathrm{C}_{70}$ requires rather harsh conditions. ${ }^{7} \mathrm{Up}$ to now, none of them have been employed as electron donors either in artificial photosynthesis or in photovoltaics.

Filling empty fullerenes with metals or metallic clusters affords endohedral metallofullerenes (EMFs), whose physical, chemical and electrochemical properties are different from those of empty fullerenes. ${ }^{8}$ For example, $\mathrm{La} @ \mathrm{C}_{82}, \mathrm{M}_{2} @ \mathrm{C}_{80}(\mathrm{M}=\mathrm{La}, \mathrm{Ce})$, and $\mathrm{M}_{3} \mathrm{~N} @ \mathrm{C}_{80}(\mathrm{M}=\mathrm{Sc}, \mathrm{Lu})$ undergo easier oxidations and feature stronger absorption throughout the visible part of the solar spectrum when compared to $\mathrm{C}_{60}$ and $\mathrm{C}_{70}$. These characteristics render EMFs p-type materials for photovoltaics. Very recently, the idea of using EMFs as electron donors has been verified in photophysical assays with $\mathrm{Lu}_{3} \mathrm{~N} @ \mathrm{C}_{80}$ or $\mathrm{La}_{2} @ \mathrm{C}_{80}$ as the electron donor and PDI or TCAQ as the electron acceptor, respectively. ${ }^{9,10}$

Subphthalocyanines (SubPcs) are aromatic chromophores, which absorb light throughout most of the visible part of the spectrum featuring (i) high extinction coefficients, (ii) excitation energies above $2.0 \mathrm{eV}$, and (iii) low reorganization energies in electron transfer reactions. ${ }^{11}$ Importantly, they do not aggregate owing to their conical shape and are known as strong fluorophores. SubPcs bearing on their periphery electron-withdrawing substituents are well-known electron acceptors. ${ }^{11 b}$ Thus, electron-deficient SubPcs have been considered as promising complements to fullerenes.

In the present work, we report on the synthesis and the properties of conjugates $\mathbf{1 a}$ and $\mathbf{1 b}$ (Scheme 1) that comprise $\mathrm{La}_{2} @ \mathrm{C}_{80}$ as electron donor and (dodecafluoro)/hexa(pentylsulfonyl)SubPc as electron acceptors. We will demonstrate that, despite negligible interactions in the ground-state, electrontransfer events occur between SubPc and $\mathrm{La}_{2} @ \mathrm{C}_{80}$ in the excited state. However, in conjugates $\mathbf{2 a}$ and $\mathbf{2} \mathbf{b}$, only energy transfer events take place. 




Scheme 1 Synthesis of $\mathrm{La}_{2} \mathrm{CC}_{80}-\mathrm{SubPc}(\mathbf{1} \mathbf{a}$ and $\mathbf{1} \mathbf{b})$ from subphthalocyanines $(\mathbf{3 a}, \mathbf{3 b})$.

Conjugates $\mathbf{1 a}, \mathbf{1 b}$ were synthesized according to the procedure previously reported for $\mathbf{2 a}{ }^{12}$ Briefly, $\mathbf{1 a}, \mathbf{1 b}$ were obtained in $25 \%$ or less via Prato reaction of $\mathrm{La}_{2} @ \mathrm{C}_{80}$ and SubPc 3a, $\mathbf{b}^{13}$ (Scheme 1). The formation of $\mathbf{1 a}$ or $\mathbf{1 b}$ as major products was revealed by HPLC and they were isolated via a multi-step HPLC procedure (Fig. S1-S3, ESI $\dagger){ }^{14}$ The compositions of $\mathbf{1 a}$ and $\mathbf{1 b}$ were confirmed using MALDI-TOF mass spectrometry (Fig. S5-S7, ESI $\dagger$ ).

To shed light onto the structural feature of $1 \mathrm{a}, \mathrm{VT}^{1} \mathrm{H}$ NMR studies were conducted. As shown in Fig. S8 (ESI $\uparrow$ ), the ${ }^{1} \mathrm{H}$ NMR spectrum measured at $283 \mathrm{~K}$ exhibits two sets of signals: those originating from pyrrolidine protons at 5-2 $\mathrm{ppm}$ and those from phenyl protons at 8-5.5 ppm, indicating the presence of at least two conformers of 1a in a 3:1 ratio. In each set, a pair of doublets, which are assigned to the geminal protons on the pyrrolidine, is discernible. Confirmation for this assignment was obtained by COSY experiments, revealing a reasonable cross-coupling relationship between the doublets (Fig. S9, ESI†). In addition, the chemical shifts as well as the chemical shift differences ( $\Delta \delta=1.2$ to $1.3 \mathrm{ppm}$ ) of these geminal protons are in a good agreement with those reported previously. ${ }^{15}$ An increase of the temperature from 283 to $313 \mathrm{~K}$ resulted only in a broadening of the NMR signals in 1a (Fig. S8, ESI $\dagger$ ) ruling out the possibility of conformational conversion in this temperature range. Characterization of $\mathbf{1 b}$ using ${ }^{1} \mathrm{H}$ NMR was, however, hampered by the small amounts of the isolated material and its poor stability.

To further investigate the structural and electronic features of 1a, DFT-calculations were performed using a Gaussian 09 package. ${ }^{16}$ Owing to the $[5,6]$-addition pattern and the unsymmetrical pyrrolidine substitution, 1a adopts at most four different conformations - Fig. S13 (ESI $\dagger$ ). Among them, conformer I, in which both, the pyrrolidine and the substituted phenoxy unit, are approaching a 5-member ring, has the lowest formation energy (Table S1, ESI $\dagger$ ) at the M06-2X/3-21G $\sim 6-31 \mathrm{G}^{*} \sim$ sdd level. ${ }^{17-19}$ In comparison, conformer II, in which both the pyrrolidine and the substituted phenoxy group are aligned close to a 6-member ring, is $2.3-3.3 \mathrm{kcal} \mathrm{mol}^{-1}$ less stable than conformer I. Conformers III and IV possess the least stability, namely $5.3 \mathrm{kcal} \mathrm{mol}^{-1}$ less than conformer I. Therefore, we hypothesize the presence of conformers I and II in purified 1a with a ratio of $3: 1$.

Electrochemical studies with 1a and 2a were carried out by means of CV and DPV. In the range from -2.0 to $1.2 \mathrm{~V}$, $2 \mathrm{a}$ reveals
Table 1 Redox potentials ${ }^{a}$ of $\mathrm{La}_{2} \mathrm{aC}_{80}-\mathrm{SubPc}$ conjugate $1 \mathrm{a}$ and reference compounds, $E_{\mathrm{p}}$ vs. Fc/Fc ${ }^{+}, \mathrm{V}$

\begin{tabular}{llllllll}
\hline & $E_{\text {ox }}^{3}$ & $E_{\text {ox }}^{2}$ & $E_{\text {ox }}^{1}$ & $E_{\text {red }}^{1}$ & $E_{\text {red }}^{2}$ & $E_{\text {red }}^{3}$ & $E_{\text {red }}^{4}$ \\
\hline $\mathbf{1 a}$ & $0.97^{b}$ & 0.58 & 0.20 & -0.47 & -1.12 & $-1.80^{b}$ & \\
$\mathbf{2 a}$ & & 1.08 & 0.99 & -1.06 & -1.18 & -1.57 & -1.79 \\
$\mathbf{4}^{c}$ & $1.00^{b}$ & 0.59 & 0.20 & -0.44 & -1.70 & -2.13 &
\end{tabular}

$4=[5,6]-$ pyrrolidine $\mathrm{La}_{2} @ \mathrm{C}_{80}: \mathrm{La}_{2} @ \mathrm{C}_{80}-\mathrm{NTrt}$ (Trt = triphenylmethyl). ${ }^{a}$ Values obtained from DPVs. ${ }^{b}$ Two-electron process. ${ }^{c}$ Data from ref. 15.

four reversible one-electron reductions at $-1.06,-1.18,-1.57$, and $-1.79 \mathrm{~V}$ and two irreversible one-electron oxidations at +0.99 and $+1.08 \mathrm{~V}$ (Table 1 and Fig. S12, ESI $\dagger$ ). The first oxidation, and the first and third reductions are $\mathrm{C}_{60}$ centered, while the second oxidation, and the second and forth reductions are centered on SubPc. As for 1a, the electrochemical patterns of the [5,6]-pyrrolidine $\mathrm{La}_{2} @ \mathrm{C}_{80}$ and perfluorinated SubPc are clearly distinguishable. ${ }^{12,15}$ In particular, the first and second reductions, which are seen as a one-electron process at -0.47 and $-1.12 \mathrm{~V}$, coincide well with the reductions of $\mathrm{La}_{2} @ \mathrm{C}_{80}$ and SubPc, respectively. The third reduction at $-1.80 \mathrm{~V}$ appears as a two-electron process, involving the second reduction of $\mathrm{La}_{2} @ \mathrm{C}_{80}$ and the second reduction of SubPc. In addition, three oxidations are visible. The first and second are fully reversible oneelectron processes, which agree well with those of $\mathrm{La}_{2} @ \mathrm{C}_{80}$. The third oxidation is a two-electron process, corresponding to the first oxidation of SubPc and the third oxidation of $\mathrm{La}_{2} @ \mathrm{C}_{80}$. Our electrochemical assays point to the fact that ground state interactions between the electroactive constituents of 1a are negligible. The remarkable oxidative features of 1a underline the strong electron donor character of $\mathrm{La}_{2} @ \mathrm{C}_{80}$, which is lacking in $\mathrm{C}_{60}$ in $2 \mathrm{a}$.

Complementary DFT-calculations further underline the electrochemical data. As shown in Fig. S14 (ESI $\dagger$ ), the calculated HOMO of 1a is mainly delocalized on $\mathrm{La}_{2} @ \mathrm{C}_{80}$, while the LUMO and $\mathrm{LUMO}+1$ are localized on the endohedral $\mathrm{La}_{2}$ cluster and on the perfluorinated SubPc, respectively.

To gain further insight into the ground state features of $\mathbf{1 a}-\mathbf{b}$, we turned to absorption spectroscopy. At a first glance, the absorption spectrum of $\mathbf{1 a - b}$ is best described as a simple superimposition of the spectra of the individual components, namely SubPc and $[5,6]$-pyrrolidine $\mathrm{La}_{2} @ \mathrm{C}_{80}$ (Fig. S10 and S11, ESI $\dagger$ ). Detailed comparison between $\mathbf{1 a}$ and $\mathbf{1 b}$ suggests that the absorption maxima of $\mathbf{1 b}$ are $4 \mathrm{~nm}$ red-shifted relative to that of 1a, thus, confirming the stronger electron-acceptor properties of sulfonated SubPcs than of the fluorinated one. Despite the presence of $[5,6]$-pyrrolidine $\mathrm{La}_{2} @ \mathrm{C}_{80}$, the absorption maximum of SubPc undergoes no shift as compared with that of SubPc $\mathbf{3 a}$ and $\mathbf{3 b}$, indicating the lack of ground-state interaction between the individual components.

In fluorescence experiments, a solvent independent fluorescence quantum yield of 0.17 was noted for $3 a$. In stark contrast, fluorescence assays with 1a point to a rather marked quenching with fluorescence quantum yields of 0.005 (toluene), 0.006 (THF), and 0.006 (benzonitrile).

To attribute our spectral observation, spectroelectrochemical experiments on $\left(\mathrm{F}_{12} \mathrm{SubPc}\right)^{\bullet-}$ and $\left(\left(\mathrm{SO}_{2} \mathrm{C}_{5} \mathrm{H}_{11}\right)_{6} \mathrm{SubPc}\right)^{\bullet-}$ as well as $\left(\mathrm{La}_{2} @ \mathrm{C}_{80}\right)^{\bullet+}$ were deemed important - Fig. S15 (ESI $\left.\dagger\right)$. On the one hand, the differential absorption spectra of the electrochemically 
reduced 3a reveal two broad features with maxima at 455 and $655 \mathrm{~nm}$, which are accompanied by shoulders at around 475 and $610 \mathrm{~nm}$, as well as a minimum at $570 \mathrm{~nm}$. Upon spectroelectrochemical reduction of $\mathbf{3 b}$, spectral characteristics including maxima at $480,545,620$, and $735 \mathrm{~nm}$ complemented by minima at 534 and $581 \mathrm{~nm}$ evolved. Notably, pulse radiolytic reductions with $\mathbf{3 a}$ or $\mathbf{3 b}$ in deaerated toluene/2-propanol/acetone mixtures $(8: 1: 1 \mathrm{v} / \mathrm{v})$ results in quantitatively similar spectra with characteristic fingerprints at 610 and $620 \mathrm{~nm}$, respectively. On the other hand, a characteristic maximum at $900 \mathrm{~nm}$ and a broad near infrared tail evolve as spectroscopic characteristics upon spectroelectrochemical oxidation of 4 .

Insights into the excited state deactivation in $\mathbf{4}, \mathbf{3 a}, \mathbf{3 b}, \mathbf{2 a}, \mathbf{2 b}, \mathbf{1 a}$, and $\mathbf{1 b}$, in general, and into the corresponding photoproducts, in particular, came from transient absorption measurements following femtosecond and nanosecond excitation. Excitation of 4 at $387 \mathrm{~nm}$ leads to the population of the $\mathrm{La}_{2} @ \mathrm{C}_{80}$ singlet excited state $(1.4 \pm$ $0.2 \mathrm{eV}$ ), which features ground state bleaching at $465 \mathrm{~nm}$ and wellresolved fine structure with maxima at 516, 466, 614, 735, 800, and $900 \mathrm{~nm}$. The latter is subject to a fast intersystem crossing $60 \pm 30 \mathrm{ps}$ - to the triplet manifold due to the presence of the $\left(\mathrm{La}_{2}\right)^{6+}$ cluster, which promotes efficient spin-orbit coupling. Following the singlet excited state decay, a weak and broad absorption in the 800-1200 $\mathrm{nm}$ region, along with broad features that taper at $550 \mathrm{~nm}$, are discernible. These features relate to the $\mathrm{La}_{2} @ \mathrm{C}_{80}$ triplet excited state $(1.0 \pm 0.1 \mathrm{eV})$.

$\mathrm{F}_{12} \mathrm{SubPc} 3 \mathrm{a}$ reveals upon excitation at $530 \mathrm{~nm}$ differential absorption changes, which include transient maxima at 440 and $600 \mathrm{~nm}$ as well as transient minima at 514, 575, and $635 \mathrm{~nm}-$ Fig. S16 (ESI $\dagger$ ). In addition, a broad near-infrared feature spans from 650 to $1200 \mathrm{~nm}$, which peaks around $710 \mathrm{~nm}$. These features relate to the singlet excited state $(2.16 \mathrm{eV})$ of $3 \mathrm{a}$, which transforms with $1.9 \pm 0.1 \mathrm{~ns}$ into the corresponding triplet excited state $(1.4 \mathrm{eV})$. Transient absorption spectra of the latter maximize at 470 and $610 \mathrm{~nm}$ and minimize at 532 and $570 \mathrm{~nm}$.

Commencing with the conclusion of the $530 \mathrm{~nm}$ excitation, SubPc 3b reveals differential absorption changes in the form of transient maxima at 424, 474, 623, $660 \mathrm{~nm}$, a broad tail extending far into the near infrared, as well as transient minima at 533 and $583 \mathrm{~nm}$ - Fig. S17 (ESI $\dagger$ ). These SubPc singlet excited state $(2.12 \mathrm{eV})$ related transient absorption features undergo intersystem crossing to the corresponding triplet excited state $(1.4 \mathrm{eV})$, which exhibits a broad transient in the visible part of the spectrum. The latter maximizes at 470 and $620 \mathrm{~nm}$ and minimizes at 533 and $583 \mathrm{~nm}$. Owing to the presence of sulfur, which facilitates spin-orbit coupling, the intersystem crossing is accelerated relative to what is seen for $3 a$ with lifetimes of $1220 \pm 20$ ps, $420 \pm 10 \mathrm{ps}, 415 \pm$ $10 \mathrm{ps}$ in toluene, THF, and benzonitrile, respectively.

Conjugate 1a gives rise upon $530 \mathrm{~nm}$ excitation to differential absorption changes in the form of transient maxima at 450,600, and $720 \mathrm{~nm}$ as well as transient minima at 575 and $635 \mathrm{~nm}$ - Fig. 1. In line with the reference experiments, namely with 3a, we assign these changes to the $\mathrm{F}_{12} \mathrm{SubPc}$ singlet exited state. Instead of seeing the slow intersystem crossing to the SubPc triplet state, the SubPc singlet excited state decays ultrafast with lifetimes of $3.0 \pm 0.4 \mathrm{ps}$ (toluene), $2.2 \pm 0.2 \mathrm{ps}$ (THF), and $2.0 \pm 0.2 \mathrm{ps}$ (benzonitrile). Simultaneously, new transitions develop in the visible and near-infrared regions.

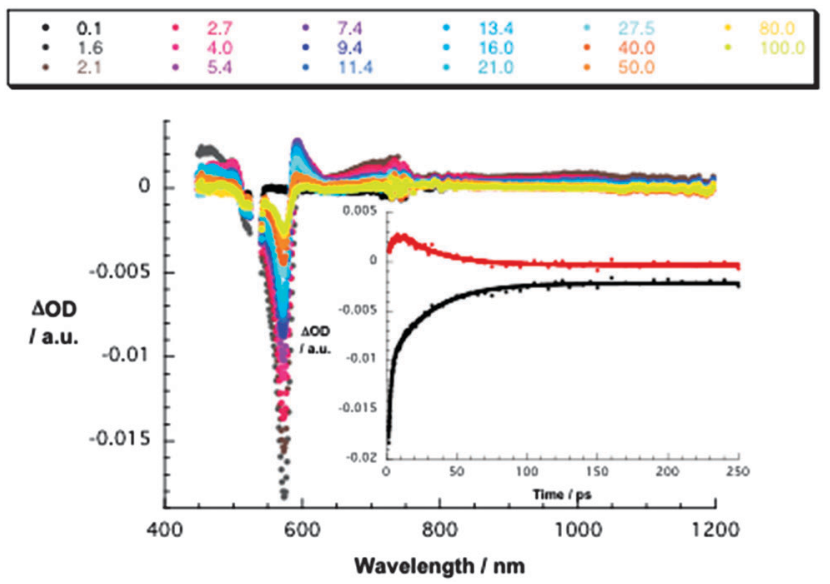

Fig. 1 Differential absorption spectra (visible and near-infrared) obtained upon femtosecond flash photolysis $(530 \mathrm{~nm})$ of $1 \mathrm{a}\left(10^{-5} \mathrm{M}\right)$ in argon-saturated THF with several time delays between 0.1 and 100 ps at room temperature. Inset: time-absorption profiles of the spectra shown at the top at 573 and $592 \mathrm{~nm}$ monitoring the charge separation and the charge recombination.

Importantly, the new transients do not match the signature of the SubPc triplet excited state. Instead, maxima at 480 and $590 \mathrm{~nm}$ as well as minima at 515 and $570 \mathrm{~nm}$ are discernible. Please note that these features bear great resemblance to the pulse radiolytic findings in the context of reducing SubPc and, as such, relate to its $\pi$-radical anion $-\left(\mathrm{F}_{12} \mathrm{SubPc}\right)^{\bullet-}$. In the nearinfrared region, a broad tail is attributable to the $\mathrm{La}_{2} @ \mathrm{C}_{80}$ $\pi$-radical cation, that is, $\left(\mathrm{La}_{2} @ \mathrm{C}_{80}\right)^{\bullet+}$. Taking the aforementioned into consideration, we conclude that an energetically low lying radical ion pair state $(1.32 \mathrm{eV})$, namely $\left(\mathrm{La}_{2} @ \mathrm{C}_{80}\right)^{\bullet+}-\left(\mathrm{F}_{12} \mathrm{SubPc}\right)^{\bullet-}$, is formed. Both fingerprints served as reliable probes to determine the lifetime of the metastable $\left(\mathrm{La}_{2} @ \mathrm{C}_{80}\right)^{\bullet+}-\left(\mathrm{F}_{12} \mathrm{SubPc}\right)^{\bullet-}$ radical ion pair state. All decays were well fitted by a single exponential fitting function throughout the femtosecond time scale. In particular, lifetimes of $34 \pm 2$ ps (toluene), $32 \pm 2 \mathrm{ps}$ (THF), and $35 \pm 2 \mathrm{ps}$ (benzonitrile) were derived.

Singlet oxygen quantum yields for 1a are as low as 0.010 in THF and support the assignment that any other state than the $\mathrm{F}_{12} \mathrm{SubPc}$ triplet excited state evolves as the product of charge recombination. Please note the singlet oxygen yields in 3a of 0.31 (toluene), 0.10 (THF), and 0.35 (benzonitrile).

Laser excitation of $\mathbf{1 b}$ in benzonitrile at $568 \mathrm{~nm}$ results immediately after excitation in differential absorption maxima at 485 and $623 \mathrm{~nm}$ and a minimum at $583 \mathrm{~nm}-$ Fig. S18 (ESI $\dagger$ ). Although these transient features relate to the singlet excited state of SubPc they decay ultrafast with a lifetime of $1.4 \pm 0.1$ ps. New transients evolve, which maximize at 480 and $610 \mathrm{~nm}$ and minimize $583 \mathrm{~nm}$. A spectral comparison with the results from the spectroelectrochemical/pulse radiolytic investigations supports the notion that the new transients are attributed to the $\pi$-radical anion $\left(\left(\mathrm{SO}_{2} \mathrm{C}_{5} \mathrm{H}_{11}\right)_{6} \mathrm{SubPc}\right)^{\bullet-}$. Taking the aforementioned into consideration, we postulate an electron transfer from $\mathrm{La}_{2} @ \mathrm{C}_{80}$ to the SubPc singlet excited state to afford $\left(\mathrm{La}_{2} @ \mathrm{C}_{80}\right)^{\bullet+}-\left(\left(\mathrm{SO}_{2} \mathrm{C}_{5} \mathrm{H}_{11}\right)_{6} \mathrm{SubPc}\right)^{\bullet-}(1.36 \mathrm{eV})$. It is worth mentioning that the detection of $\left(\mathrm{La}_{2} @ \mathrm{C}_{80}\right)^{\bullet+}$ in the near infrared is hampered by the thermal decomposition of SubPc. This is seen to form a product with absorptions in the $700-850 \mathrm{~nm}$ range. 


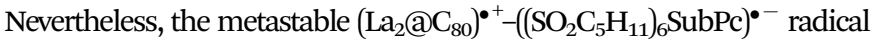
ion pair state decays with $28 \pm 2$ ps to the singlet ground state.

Likewise, $530 \mathrm{~nm}$ excitation of $\mathrm{F}_{12} \mathrm{SubPc}_{60} 2 \mathrm{a}$ results in the exclusive formation of the SubPc singlet excited state - Fig. S19 (ESI $\dagger$ ). In particular, transient maxima at 450,600 , and $720 \mathrm{~nm}$ as well as transient minima at 515, 575, and $635 \mathrm{~nm}$ are formed and decay rapidly with $1.5 \pm 0.3 \mathrm{ps}$ (toluene), $1.5 \pm 0.3 \mathrm{ps}$ (THF), and $1.4 \pm$ $0.3 \mathrm{ps}$ (benzonitrile). As the SubPc singlet excited state decay comes to an end a broad near-infrared transient, which maximizes at $910 \mathrm{~nm}$, is noted, suggesting a $\mathrm{C}_{60}$ singlet excited state. Interestingly, we did not find the characteristic $\mathrm{C}_{60}$ triplet feature at $700 \mathrm{~nm}$ at the end of the $\mathrm{C}_{60}$ singlet excited state deactivation. In contrast, maxima at 470 and $615 \mathrm{~nm}$ as well as a minimum at $575 \mathrm{~nm}$ were found, pointing to the SubPc triplet excited state. ${ }^{12}$ From this we infer that the $\mathrm{C}_{60}$ triplet excited state $(1.5 \mathrm{eV})$ undergoes a thermodynamically allowed transfer of triplet excited state energy to SubPc $(1.4 \mathrm{eV})$. The kinetics at the 470 and $615 \mathrm{~nm}$ maxima further furnishes the kinetic assignment, namely the rate-determining step in the SubPc triplet excited state formation is the $\mathrm{C}_{60}$ centered intersystem crossing. A global analysis reveals kinetics that are very similar $(1.6 \pm 0.1 \mathrm{~ns})$ to that of the inherent intersystem crossing dynamics seen for $\mathrm{C}_{60}$. In this context, it is reassuring that the transients seen at the end of the femtosecond experiments matches that at the beginning of the nanosecond experiment. Moreover, maxima at 470 and $610 \mathrm{~nm}$, minima at 532 and $570 \mathrm{~nm}$, and an excited state lifetime of $36 \mu$ s without oxygen perfectly agree with the SubPc triplet excited state of 3a. Likewise, singlet oxygen quantum yields of $2 \mathbf{a}$ were found to be as high as 0.28 (toluene), 0.13 (THF), and 0.41 (benzonitrile) and support the assignment that the triplet excited state evolves as the product of charge recombination. Please note that the singlet oxygen yields in $\mathbf{3 a}$ are as high as 0.35 .

When turning to $\mathbf{2 b}$, excitation at $530 \mathrm{~nm}$ is accompanied by the formation of its singlet excited state - Fig. S20 (ESI $†$ ). Evidence stems from monitoring maxima at $427,474,623$, and $660 \mathrm{~nm}$ and minima at 535 and $585 \mathrm{~nm}$. These decay in the presence of $\mathrm{C}_{60}$ rapidly with $1.5 \pm 0.3 \mathrm{ps}$ (toluene), $1.5 \pm 0.3 \mathrm{ps}$ (THF), and $1.4 \pm 0.3 \mathrm{ps}$ (benzonitrile) to form accordingly the $\mathrm{C}_{60}$ singlet excited state with its $910 \mathrm{~nm}$ maximum. Like for 2a, we did not find the characteristic $\mathrm{C}_{60}$ triplet feature. Instead, maxima at 470 and $625 \mathrm{~nm}$ as well as minima at 535 and $585 \mathrm{~nm}$ of the SubPc triplet excited state were concluded. In other words, the triplet excited state of SubPc (1.4 eV) evolves from a thermodynamically allowed transfer of triplet excited state. The kinetics at the 470 and $625 \mathrm{~nm}$ maxima and the $585 \mathrm{~nm}$ minimum document that the rate-determining step is the $\mathrm{C}_{60}$ centered intersystem crossing ( $1.6 \pm 0.1 \mathrm{~ns})$. In the absence of oxygen, the SubPc triplet excited state lifetime is $20 \pm 5 \mu$ s in agreement with what was found for $\mathbf{3 b}$.

Electron accepting SubPcs have been used in combination with $\mathrm{La}_{2} @ \mathrm{C}_{80}$ to prepare a series of novel $\mathrm{La}_{2} @ \mathrm{C}_{80}-\mathrm{SubPc}$ electron donor-acceptor conjugates to mimic the photosynthetic apparatus. Our results in terms of electrochemical and steady-state absorption reveal no appreciable electronic interactions between SubPc and $\mathrm{La}_{2} @ \mathrm{C}_{80}$ in the ground state. This changed in the excited state, where an intramolecular electron-transfer evolves from $\mathrm{La}_{2} @ \mathrm{C}_{80}$ to photoexcited SubPc. In comparison, reference conjugates of $\mathrm{C}_{60}$ and
SubPcs feature only a singlet-singlet energy transfer from SubPc to $\mathrm{C}_{60}$. Thus, replacing $\mathrm{C}_{60}$ by $\mathrm{La}_{2} @ \mathrm{C}_{80}$ provides a promising way to tune energy-transfer versus electron-transfer. Furthermore, considering the short separations between the electron donors and acceptors, optimizing the charge-separated state lifetimes seems achievable via the tailored design of linkers between $\mathrm{La}_{2} @ \mathrm{C}_{80}$ and SubPc.

This work is financially supported by the NSFC (51372158), the NSF of Jiangsu Province (BK2012611), the Jiangsu Specially Appointed Professor Program (SR10800113), Priority Academic Program Development of Jiangsu Higher Education Institutions (PAPD), the Project for Jiangsu Scientific and Technological Innovation Team (2013), Spanish MEC and MICINN (CTQ2011-24187/BQU and PRI-PIBUS-2011-1128), the Deutsche Forschungsgemeinschaft (GU 517/14-1) and the Comunidad de Madrid (S2013/MIT-2841, FOTOCARBON).

\section{Notes and references}

1 A. A. Boghossian, M. H. Ham, J. H. Choi and M. S. Strano, Energy Environ. Sci., 2011, 4, 3834-3843.

2 (a) S. Hammes-Schiffer, Acc. Chem. Res., 2009, 42, 1881-1889; (b) M. R. Wasielewski, Chem. Rev., 1992, 92, 435-461.

3 G. de la Torre, G. Bottari, M. Sekita, A. Hausmann, D. M. Guldi and T. Torres, Chem. Soc. Rev., 2013, 42, 8049-8105.

4 G. Bottari, G. de la Torre, D. M. Guldi and T. Torres, Chem. Rev., 2010, 110, 6768-6816.

5 S. Kirner, M. Sekita and D. M. Guldi, Adv. Mater., 2014, 26, 1482-1493.

6 (a) J. L. Delgado, P. A. Bouit, S. Filippone, M. A. Herranz and N. Martín, Chem. Commun., 2010, 46, 4853-4865; (b) D. M. Guldi, Chem. Soc. Rev., 2002, 31, 22-36.

7 (a) K. Ohkubo, J. Ortiz, L. Martin-Gomis, F. Fernandez-Lazaro, A. Sastre-Santos and S. Fukuzumi, Chem. Commun., 2007, 589-591; (b) C. A. Reed, K. C. Kim, R. D. Bolskar and L. J. Mueller, Science, 2000, 289, 101-104; (c) S. Fukuzumi, H. Mori, H. Imahori, T. Suenobu, Y. Araki, O. Ito and K. M. J. Kadish, J. Am. Chem. Soc., 2001, 123, 12458-12465.

8 (a) A. A. Popov, S. Yang and L. Dunsch, Chem. Rev., 2013, 113, 5989-6113; (b) X. Lu, L. Feng, T. Akasaka and S. Nagase, Chem. Soc. Rev., 2012, 41, 7723-7760.

9 (a) M. Rudolf, L. Feng, Z. Slanina, T. Akasaka, S. Nagase and D. M. Guldi, J. Am. Chem. Soc., 2013, 135, 11165-11174; (b) L. Feng, M. Rudolf, S. Wolfrum, A. Troeger, Z. Slanina, T. Akasaka, S. Nagase, N. Martín, T. Ameri, C. J. Brabec and D. M. Guldi, J. Am. Chem. Soc., 2012, 134, 12190-12197.

10 Y. Takano, S. Obuchi, N. Mizorogi, R. García, M. A. Herranz, M. Rudolf, D. M. Guldi, N. Martín, S. Nagase and T. Akasaka, J. Am. Chem. Soc., 2012, 134, 19401-19408.

11 (a) C. G. Claessens, D. Gonzalez-Rodriguez, M. S. Rodriguez-Morgade, A. Medina and T. Torres, Chem. Rev., 2014, 114, 2192-2277; (b) C. Romero-Nieto, A. Medina, A. Molina-Ontoria, C. G. Claessens, L. Echegoyen, N. Martin, T. Torres and D. M. Guldi, Chem. Commun., 2012, 48, 4953-4955.

12 D. Gonzalez-Rodriguez, T. Torres, D. M. Guldi, J. Rivera, M. A. Herranz and L. Echegoyen, J. Am. Chem. Soc., 2004, 126, 6301-6313.

13 M. Ince, A. Medina, J. H. Yum, A. Yella, C. G. Claessens, M. V. Martinez-Diaz, M. Graetzel, M. K. Nazeeruddin and T. Torres, Chem. Eur. J., 2014, 20, 2016-2021.

14 In the reaction mixture, besides $1 \mathrm{a}$, a minor product $\mathbf{1 a}^{\prime}$ was also isolated and identified as a [6,6]-pyrrolidine adduct using MALDITOF mass and UV-vis-NIR absorption spectra (Fig. S6 and S9, ESI $\dagger$ ).

15 (a) M. Yamada, M. Okamura, S. Sato, I. C. Someya, N. Mizorogi, T. Tsuchiya, T. Akasaka, T. Kato and S. Nagase, Chem. - Eur. J., 2009, 15, 10533-10542; (b) T. Cai, Z. Ge, E. B. Lezzi, T. E. Glass, K. Harich, H. W. Gibson and H. C. Dorn, Chem. Commun., 2005, 3594-3596.

16 M. J. Frisch, et al., GAUSSIAN 09, Revision A. 02, Gaussian Inc., Wallingford, CT, 2009.

17 Y. Zhao and D. G. Truhlar, Theor. Chem. Acc., 2008, 120, 215-241.

18 X. Y. Cao and M. Dolg, THEOCHEM, 2002, 581, 139-147.

19 A. D. Becke, J. Chem. Phys., 1993, 98, 5648-5652. 